\title{
Conceptualización de los Estudiantes sobre el Buen Profesor Universitario en las Carreras de la Salud de la Universidad de La Frontera - Chile
}

\author{
Concept of Good University Teacher in Students of Health Careers, \\ of the Universidad de La Frontera-Chile \\ *Daisy Cabalín Silva \& **Nancy Navarro Hernández
}

\begin{abstract}
CABALÍN, S. D. \& NAVARRO, H. N. Conceptualización de los estudiantes sobre el buen profesor universitario en las Carreras de la Salud de la Universidad de La Frontera-Chile. Int. J. Morphol., 26(4):887-892, 2008.

RESUMEN: La tendencia educativa de centrar los procesos en el estudiante, implica un cambio de rol en el profesorado y desarrollo de nuevas competencias; indagarlas resulta relevante. Para ello se planteó un estudio cualitativo con el propósito de conocer la representación del concepto "Buen profesor universitario", que tienen los estudiantes de primer año de las Carreras de la Salud de la Facultad de Medicina de la Universidad de La Frontera, en base a la técnica de redes semánticas naturales. La muestra estuvo constituida por 277 estudiantes de las carreras de Enfermería (43), Kinesiología (38), Medicina (53), Nutrición (30), Odontología (57), Obstetricia (24) y Tecnología Médica (32). En el análisis de datos se obtuvieron los valores J, M, Conjunto SAM y FMG propios de la técnica mencionada. Se identificaron en el núcleo de la red semántica, tres grupos de palabras que corresponden a conceptos relacionados con atributos valóricos, de relaciones interpersonales, de responsabilidad y de cómo el profesorado desarrolla y enfrenta la práctica educativa. Los estudiantes valoran principalmente el desarrollo de competencias genéricas y actitudes personales que se relacionan con el deber ser y «saber convivir» en el profesorado, lo que implica para el docente el reconocerlas, valorarlas y desarrollarlas para enfrentar de mejor forma los cambios educativos, contribuyendo a una formación integral de los futuros profesionales de la salud.
\end{abstract}

PALABRAS CLAVE: Profesor universitario; Redes semánticas; Competencias.

\section{INTRODUCCIÓN}

Educadores, estudiantes e instituciones educativas son el eje fundamental para la formación humana, la que ha sufrido cambios, ayudada en gran medida por la creciente generación de conocimiento, los avances tecnológicos, culturales y sociales.

Actualmente, la tendencia de los procesos educativos es cambiar la concepción de la enseñanza y aprendizaje, de un paradigma centrado en el profesor a uno centrado en el estudiante, de la transmisión de conocimientos, a la construcción de éste, valorando la importancia no sólo de los resultados de aprendizajes, sino de los procesos inherentes para su construcción; donde no está instaurada una relación unidireccional en cuanto a la transmisión de conocimiento, sino mas bien existe un proceso de interrelación entre el educador y el educando sobre la base de las experiencias desa- rrolladas, favoreciendo los cuatro pilares fundamentales de la educación "aprender a conocer", "aprender a hacer", "aprender a convivir y "aprender a ser" (Delors 1996). Cabe destacar que en este proceso se desarrollan, además de la capacidad intelectual, la propia personalidad de los educandos, se promueven valores, modos de conceptuar la realidad, motivaciones y cuestiones éticas que inciden en la conducta social.

Por otra parte, las exigencias actuales de la sociedad han demostrado que la creatividad, el ser aprendedor de por vida, la capacidad de liderazgo, la capacidad crítica y autocrítica, trabajo en equipo, habilidades interpersonales, la búsqueda de información, la resolución de problemas, la complejidad para abordar la problemática de salud, la reflexión en la acción son, entre otras, competencias indis-

\footnotetext{
Profesor de Estado, Magíster Pedagogía y Gestión Universitaria. Oficina de Educación en Ciencias de la Salud (OFECS). Facultad de Medicina, Universidad de La Frontera. dcabalin@ufro.cl Casilla 54 D Temuco, Chile.

** Matrona, Magíster Pedagogía y Gestión Universitaria. Oficina de Educación en Ciencias de la Salud (OFECS). Facultad de Medicina, Universidad de La Frontera. nnavarro@ufro.cl Casilla 54 D Temuco, Chile.
} 
pensables para el desempeño laboral de los futuros profesionales de la salud (Tunning Educational Structures in Europe, 2003).

Ya en la década del 70, Freire (1970) planteaba que la educación se debe basar en la manera como el alumno ve y siente el mundo a su alrededor, cuestionando a la educación tradicional de su época, donde el profesor lo sabe y lo puede todo, mientras que el alumno no sabe nada y es totalmente dependiente.

En el mismo sentido, Morín (1999) y Figueroa et al. (1981) coinciden en replantear el propósito de la educación desde una perspectiva más humana, la cual debe estar orientada a contribuir al desarrollo integral de los actores involucrados en el proceso socioeducativo. Para González (2000), no sólo se aprenden conocimientos y habilidades, sino también valores y sentimientos, que se expresan en la conducta del ser humano como motivos de actuación, donde el profesor es un guía que conduce al estudiante por el camino del saber, estableciendo relaciones afectivas basadas en la aceptación, el respeto mutuo y la comprensión.

En esta tendencia educativa, nace una nueva concepción del profesor universitario, donde Santos (1990) considera que éste "ha de ser un conocedor de la disciplina que desarrolla, un especialista en el campo del saber, permanentemente abierto a la investigación y a la actualización del conocimiento. Pero ha de saber, también, qué es lo que sucede en el aula, cómo aprenden los alumnos, cómo se pueden organizar para ello el espacio y el tiempo, qué estrategias de intervención pueden ser más oportunas en ese determinado contexto". El profesor, pues, no es sólo un experto conocedor de una disciplina sino un especialista en el diseño, desarrollo, análisis y evaluación de su propia práctica educativa.

Por lo tanto, el profesor además de poseer las competencias de un saber científico, debe poseer las competencias que le exige la tarea docente. Tradicionalmente, la interpretación más común del término docencia es la que hace referencia a la enseñanza, a la transmisión de conocimientos culturales y científicos Benedito (1983). En este sentido, será la actividad del docente dirigida a producir estos efectos.

Por otra parte, Cervantes (1999) plantea que la labor del académico de hoy, se dirige hacia la conformación de una actitud propositiva y multidisciplinaria, capaz de obtener las conexiones entre las disciplinas, fomentar una participación más cercana hacia los problemas de su entorno y estar abierto a las críticas que puedan mejorar sus aportes científicos. Este proceso supone una interacción entre profesor y estudiante, donde las acciones que se llevan a cabo son acciones comunicativas, por tanto, según Angulo (1994), intervienen los deseos, intereses, motivaciones y expectativas de los involucrados.

Sin embargo, el profesor universitario, según De Miguel (apud García 2001 op cit), está demasiado imbuido en su rol profesional o científico de una disciplina y desde ese rol intenta ejercer su acción docente, lo que conlleva a asociar el concepto de "buen profesor" al de un "buen investigador".

Algunos estudios señalan ciertas características de los docentes, según Das (1996), para los académicos más tradicionales, un buen docente es quien; "explica la materia con claridad", "presenta la materia ordenada", "posee un buen dominio del tema", "está actualizado". Otras características son reportadas en el estudio de Cataldi (2004), donde se identifican cinco aspectos que los estudiantes desean de sus docentes: "que sea justo", "que sea paciente", "que sea claro en sus explicaciones", "que explique las veces que sea necesario", "que se interese por sus alumnos". Por otra parte, Snadden (1996) menciona que para los estudiantes un buen docente es aquel que: "genera un clima de confianza", "permite la participación activa", "demuestra interés en el aprendizaje de sus alumnos", "exhibe una orientación humanista" y "entrega retroalimentación positiva", todos ellos atributos en el ámbito del saber ser, saber actuar, saber convivir.

Si la tendencia de los procesos educativos es cambiar la concepción de un paradigma centrado en el profesor a uno centrado en el estudiante, necesariamente esto implica un cambio de rol del profesorado universitario y el desarrollo de competencias que respondan a estas nuevas exigencias. ¿Esto será percibido por los estudiantes?. ¿Cuáles serán los atributos o cualidades de un buen profesor universitario, desde la perspectiva del estudiantado?

Frente a estas interrogantes, se plantea una investigación con enfoque cualitativo en base a la técnica de redes semánticas naturales, con el objetivo de conocer las conceptualizaciones sobre el "Buen Profesor Universitario", que tienen los estudiantes de primer año de las Carreras de la Salud de la Facultad de Medicina de la Universidad de La Frontera, a modo de indagar sobre la percepción que ellos tienen al iniciar su formación universitaria.

La técnica de redes semánticas, ofrece un medio empírico de acceso a la organización cognitiva del conocimiento. Por tanto, puede proporcionar datos referentes a la organización e interpretación interna de los significantes. También indica como la información, fue percibida individualmente en el curso de la composición del aprendizaje social y provee indicios fundamentales a cerca de la tenden- 
cia a actuar basándose en ese "universo cognitivo" (Valdés \& Reyes, 1992). Estas redes semánticas se basan en las estructuras cognitivas, que describen los procesos psicológicos, desarrollados en la obtención, uso, almacenamiento y modificación del conocimiento, acerca del mundo y de las personas. Figueroa, plantea que la información almacenada está organizada en forma de redes, en las cuales las palabras, eventos o representaciones, forman relaciones que en conjunto producen significados.

Por otra parte, Morales et al. (1994) señalan que los procesos sociales cognitivos, explican la percepción y la conducta como una reacción al significado psicológico de la situación, mediada por el funcionamiento cognitivo del individuo, en virtud del cual, se da sentido al mundo de los estímulos y no por simples aprendizajes o instintos. Concluyen que la memoria semántica toma en cuenta la capacidad humana para construir la realidad, en una interpretación interna y es a través de ésta, que se decodifican las experiencias pasadas, predicciones y causalidades, las que se conectan dentro de combinaciones nuevas.

Los resultados del estudio permitirán al profesorado reflexionar sobre los conceptos del "Buen Profesor Universitario"que los estudiantes consideran como los más importantes, como asímismo, en relación a las competencias y características que el docente deberá asumir frente a las nuevas concepciones del proceso enseñanza-aprendizaje, de tal forma de contribuir a mejorar la calidad de la formación de los profesionales de la salud.

\section{METODOLOGÍA}

Para obtener la representación del concepto "Buen Profesor Universitario" desde la perspectiva de los estudiantes en base a la técnica de redes semánticas naturales, se aplicó el instrumento a los estudiantes de primer año de la Facultad de Medicina al finalizar el primer semestre académico, a fin de conocer las conceptualizaciones que ellos tienen al ingresar a la Educación Superior.

Se solicitó a los estudiantes que frente a las palabras estímulo: "Buen Profesor Universitario", expresaran con diez palabras definidoras el concepto, a su vez las jerarquizaran de acuerdo a la importancia que ellos le asignen, siendo el número uno la palabra que mejor lo define y el número diez el que más se aleja de ella. Otorgando para ello, no más de cinco minutos para la primera tarea y no más de dos minutos para la segunda (Valdéz, 1998).

La muestra estuvo constituida por 277 estudiantes
(71\% ingreso 2006) del primer año de las Carreras del área de la Salud, impartidas en la Facultad de Medicina de la Universidad de La Frontera: Enfermería (43), Kinesiología (38), Medicina (53), Nutrición (30), Odontología (57), Obstetricia (24) y Tecnología Médica (32), quienes fueron invitados a participar, informándoles del propósito del estudio, solicitando su consentimiento, garantizando el anonimato y la confidencialidad de los datos.

Para el análisis de las redes semánticas naturales se consideraron los indicadores fundamentales descritos por Figueroa; Lagunes (1993); Valdez (1998): tamaño de la red (TR) "valor J", peso semántico "valor M", conjunto SAM y valor FMG.

- Tamaño de la red (TR), "valor J": total de las palabras definidoras que fueron generadas por los sujetos para definir la palabra estímulo.

- Peso semántico, "valor M": valor obtenido de la multiplicación entre la frecuencia de aparición por la jerarquía obtenida, para cada una de las palabras definidoras generadas.

- Conjunto SAM: grupo de las diez palabras definidoras que obtienen los mayores "valores M".

- Valor FMG: valor obtenido para todas las palabras definidoras que conforman el conjunto SAM, en términos de porcentajes, de la distancia semántica que hay entre las diferentes palabras definidoras, tomando la palabra con mayor peso semántico como referencia del 100\%.

El criterio de credibilidad del estudio se garantizó mediante la triangulación de investigadores y la evidencia de los procedimientos realizados.

\section{RESULTADOS}

Los 277 estudiantes de las Carreras de la Salud de la Facultad de Medicina, emitieron 260 palabras definidoras para la palabra estímulo "Buen Profesor Universitario", con un promedio de ocho palabras por encuesta, resultando un conjunto SAM por las diez palabras con mayor peso semántico (Valor M), presentado en laTabla I.

Al graficar el conjunto SAM, claramente se pueden identificar tres grupos de palabras, que son señalados en la Fig. 1.

El primer grupo de palabras corresponde a las palabras "Respetuoso" y "Responsable" con los mayores por- 
centajes cercanos al $100 \%$ del conjunto SAM. Entre el $53 \%$ y $38 \%$ se encuentra el segundo grupo, descrito por los conceptos "Comprensivo", "Empático", "Puntual", "Inteligente” y "Amable". Y el tercer grupo lo conforman las palabras con un porcentaje alrededor del 30\%: "Claro", "Organizado" y "Motivador". Los significados colectivos de los conceptos, en la medida que la distancia semántica se aleja de la frase estímulo, da lugar a palabras definidoras con significados más individuales que colectivos estando aún dentro de la red central.

Tabla I Conjunto SAM del concepto "Buen Profesor Universitario" de los estudiantes de primer año de las Carreras de la Salud de la Universidad de La Frontera, Chile.

\begin{tabular}{llcc}
\hline $\mathbf{N}^{\mathbf{2}}$ & \multicolumn{1}{c}{$\begin{array}{c}\text { Palabras } \\
\text { definidoras }\end{array}$} & $\begin{array}{c}\text { Peso } \\
\text { semántico } \\
\text { Valor } \mathbf{M}\end{array}$ & $\begin{array}{c}\text { Valor } \\
\text { FMG } \\
\text { \% }\end{array}$ \\
\hline 1 & Respetuoso & 1.115 & 100,0 \\
2 & Responsable & 1.094 & 98,1 \\
3 & Comprensivo & 595 & 53,4 \\
4 & Empático & 534 & 47,9 \\
5 & Puntual & 460 & 41,3 \\
6 & Inteligente & 441 & 39,6 \\
7 & Amable & 423 & 38,0 \\
8 & Claro & 329 & 29,5 \\
9 & Organizado & 317 & 28,4 \\
10 & Motivador & 310 & 27,8 \\
\hline
\end{tabular}

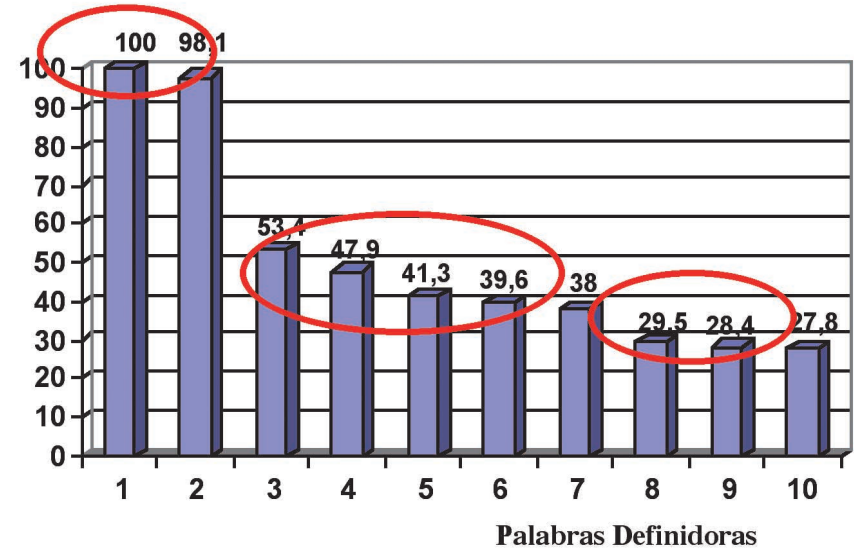

Fig. 1. Representación del núcleo semántico de la red.

Con el fin de buscar similitudes y diferencias entre las opiniones de los estudiantes de las diferentes Carreras, se muestran en la Tabla II, el número de palabras definidoras por Carrera, las tres palabras definidoras con mayor peso semántico y el valor FMG.

Se observa que para todas las Carreras, las palabras definidoras con mayor peso semántico tienen relación con atributos valóricos, "Respetuoso" y "Responsable", exceptuando la Carrera de Enfermería que menciona la palabra "Conocimiento" con mayor peso semántico.

Tabla II. Palabras definidoras del "Buen Profesor Universitario" por Carrera.

\begin{tabular}{lclcc}
\hline Carrera & $\begin{array}{c}\mathbf{N}^{\mathbf{0}} \text { Palabras } \\
\text { definidoras }\end{array}$ & $\begin{array}{c}\text { Palabras } \\
\text { definidoras }\end{array}$ & $\begin{array}{c}\text { Peso } \\
\text { semántico }\end{array}$ & $\begin{array}{c}\text { Valor FMG } \\
\text { \% }\end{array}$ \\
\hline Enfermería & 84 & Conocimiento & 202 & 100,0 \\
& & Respetuoso & 155 & 76,7 \\
Kinesiología & \multirow{2}{*}{92} & Responsable & 109 & 53,9 \\
& & Responsable & 167 & 100,0 \\
Medicina & & Respetuoso & 137 & 82,0 \\
& \multirow{2}{*}{119} & Empático & 104 & 62,3 \\
Nutrición & Responsable & 206 & 100,0 \\
& & Respetuoso & 202 & 90,1 \\
& \multirow{2}{*}{95} & Empático & 170 & 84,5 \\
Obstetricia & Responsable & 139 & 100,0 \\
& & Respetuoso & 108 & 67,7 \\
& \multirow{2}{*}{56} & Organizado & 88 & 61,2 \\
Odontología & Respetuoso & 93 & 100,0 \\
& & Responsable & 71 & 81,7 \\
& \multirow{2}{*}{123} & Empático & 72 & 77,4 \\
Tec. Médica & Respetuoso & 265 & 100,0 \\
& & Responsable & 149 & 55,6 \\
& \multirow{2}{*}{139} & Organizado & 116 & 43,3 \\
& & Responsable & 129 & 100,0 \\
& & Respetuoso & 84 & 65,1 \\
& & Amable & 48 & 37,2 \\
\hline
\end{tabular}




\section{DISCUSIÓN}

Para los estudiantes de primer año de la Facultad de Medicina de la Universidad de La Frontera, el "Buen Profesor Universitario" principalmente es definido por atributos valóricos (respetuoso y responsable). Ellos destacan como segundo grupo de palabras (comprensivo, empático, puntual, inteligente, amable), todas ellas son características personales del docente que favorecen las interrelaciones que se establecen entre docentes y estudiantes en todo proceso enseñanza aprendizaje, así como el tercer grupo (claro, organizado, motivador), que da cuenta del cómo un docente debería enfrentar la práctica educativa.

Esto refleja la importancia que los estudiantes le confieren al desarrollo de competencias genéricas interpersonales, definidas en el Proyecto Tunning )Tunning Educational Structures in Europe) y al desarrollo de "actitudes personales" que se relacionan con "el deber ser" y "saber convivir" de los aprendizajes fundamentales de Delors. De lo expresado por los estudiantes, se infiere que el profesorado debería poseer dichas competencias, lo que contribuiría a una relación didáctica armónica, para el logro de los objetivos formativos de los estudiantes, en un espacio social de comunicación e intercambio dinámico, como lo define Angulo.
Los resultados son concordantes con lo expresado por González, en relación a los procesos de formación, donde el profesor es un guía que conduce al estudiante por el camino del saber, estableciendo relaciones afectivas basadas en la aceptación, respeto mutuo y comprensión.

Llama la atención que los estudiantes no hacen referencia a características relacionadas al conocimiento disciplinar, ni relativas al manejo pedagógico de los docentes. Fundamentan sus necesidades, básicamente desde un aspecto social y habilidades interpersonales, en relación a lo que esperan de las características del profesor universitario. Podría ser que ellos asuman que los docentes poseen el dominio disciplinar y pedagógico o realmente no lo visualizan como importante en este nivel de formación.

Mejorar la calidad de la formación de los profesionales de la salud, pasa necesariamente por las competencias que posean los docentes en desempeñar su rol. Identificarlas, valorarlas y desarrollarlas constituye todo un desafío para enfrentar las nuevas tendencias educacionales que implican un cambio en la conducción de los procesos educativos.

CABALÍN, S. D. \& NAVARRO, H. N. Concept of good university teacher in students of health careers of the Universidad de La Frontera-Chile. Int. J. Morphol., 26(4):887-892, 2008.

SUMMARY: The educational tendency to focus processes on the student, implies a change in teacher's role, as well as the development of new competences, so research on the subject becomes relevant. With this need in mind, a qualitative study was proposed, in order to know how the students of first year of health careers of Facultad de Medicina de la Universidad de La Frontera represent the concept "Good university teacher" using the natural semantic networks technique. The sample were 277 students of careers of Nursing (43), Kinesiology (38), Medicine (53), Nutrition (30), Odontology (57), Midwifery (24) and Medical Technology (32). At analyzing data , the characteristic values of J, M, SAM set, and FMG were found. In the semantic network core three groups of word were identified, corresponding to concepts related to personal value attributes: interpersonal relationships, reliability, and how the teachers develop and face educational praxis. Students give a high value to the development of generic competences and personal attitudes related to how the teachers should be, how teachers should relate to others. For teachers, this means identify them, give them value, and develop them in order to face in a better way the changes in education, so contributing to the integral development of future health professionals.

KEY WORDS: University professor; Semantic networks; Competencies.

\section{REFERENCIAS BIBLIOGRÁFICAS}

Angulo, F. Enfoque práctico del currículo. En Angulo, F., Blanco L. Teoría y desarrollo del currículo. Málaga, Aljibe, 1994.

Benedito V. La docencia en la universidad. Cualidades, formación y evaluación del profesor universitario. Revista Española Investigaciones Sociológicas; 24:143-61, 1983.
Cataldi, Z. Un nuevo perfil del profesor universitario. Revista de informática educativa y medios audiovisuales, 1(3):28-33, 2004.

Cervantes, E. La Valoración del Trabajo Académico: Cómo Evaluar al Profesorado. Sociológica. UNAM-México D. F., 14 (41):223-9, 1999. 
Das M. Student y faculty perceptions of the characteristics o fan ideal teacher in a classroom setting. Medical Teacher, 18:141-6, 1996.

Delors J. La educación encierra un tesoro. Informe de la UNESCO de la Comisión Internacional sobre la Educación para el Siglo XXI. Barcelona, Grupo Santillana, 1996.

Freire, P. Pedagogía del Oprimido. $14^{\mathrm{a}}$ ed. México, Siglo Veintiuno, 1970.

Figueroa, J.; González, E. \& Solís, V. Una aproximación al problema del significado: las redes semánticas. Revista Latinoamericana de Psicología; 13(3):447-58, 1981.

García A. et al. La función docente del profesor universitario, su formación y desarrollo profesional. Didáctica Universitaria. Madrid, La Muralla, 2001.

González, V. La Profesionalidad del Docente Universitario desde una Perspectiva Humanista de la Educación. Ponencia presentada en el I Congreso Iberoamericano de Formación de Profesores, Universidad Federal de Santa María, Río Grande del Sur, Brasil. http:// www.campus-oei.org/valores/gonzalezmaura.htm., 2000.

Lagunes, I. Las redes semánticas naturales, su conceptualización y su utilización en la construcción de instrumentos. Revista de Psicología Social y Personalidad, 11:81-97, 1993.

Morales et al. Un Modelo Psicoeducativo para la Formación Valórico-Actitudinal, en Agora. (Revista del Centro Regional de Investigación Humanística, Económica y Social-CRIHES),Universidad de Los Andes, Núcleo Universitario Rafael Rangel, Consejo de Desarrollo Científico, Humanístico y Tecnológico (CDCHT). Trujillo, 4, 87-110, 1994

Morín, E. Los Siete Saberes Necesarios a la Educación del Futuro. Organización de las Naciones Unidas para la Educación, la Ciencia y la Cultura, 1999.

Snadden D. General practice and medical education: what do medical students value?. Medical Teacher, 18:31-4, 1996.

Santos, M. A. Criterios de referencia sobre calidad del proceso de enseñanza/aprendizaje en la universidad. Primeras Jornadas Nacionales de Didáctica Universitaria, Madrid, Consejo de Universidades, 1990.
Tuning Educational Structures in Europe. Informe Final. Universidad de Deusto - Universidad de Groningen. Edición y Cultura. Sócrates, 2003. pp.81-4.

Valdez, J. L. Las redes semánticas naturales, usos y aplicaciones en psicología social. México, Universidad Autónoma del Estado de México, 1998.

Valdez, J. L. \& Reyes L. Las categorías semánticas y el autoconcepto. Rev. Psicología Social en México. IV:1939, 1992.

Dirección para correspondencia:

Prof. Daisy Cabalín Silva

Oficina de Educación Médica

Facultad de Medicina

Universidad de La Frontera

Casilla 54-D

Temuco

CHILE

Email: dcabalin@ufro.cl

Received: 01-09-2008

Accepted: 02-10-2008 